\title{
Oscillation for a Class of Fractional Differential Equation
}

\author{
Qian Feng, Anping Liu \\ School of Mathematics and Physics, China University of Geosciences, Wuhan, China \\ Email:1610820876@qq.com
}

How to cite this paper: Feng, Q. and Liu, A.P. (2019) Oscillation for a Class of Fractional Differential Equation. Journal of $A p$ plied Mathematics and Physics, 7, 14291439.

https://doi.org/10.4236/jamp.2019.77096

Received: May 25, 2019

Accepted: July 3, 2019

Published: July 10, 2019

\begin{abstract}
We consider the oscillation of a class fractional differential equation with Robin and Dirichlet boundary conditions. By generalized Riccati transformation technique and the differential inequality method, oscillation criteria for a class of nonlinear fractional differential equation are obtained.
\end{abstract}

\section{Keywords}

Oscillation, Fractional Derivative, Fractional Partial Differential Equation

\section{Introduction}

The fractional differential equations are used to describe mathematical models of numerous real processes and phenomena studied in many areas of science and engineering such as population dynamics, neural networks, industrial robotics, electric circuits, optimal control, biotechnology, economics and many other branches of science. Furthermore, the fractional calculus can also provide an excellent instrument for the description of memory and hereditary properties of various materials and processes due to the existence of a "memory" term in the model.

The oscillation theory as a part of the qualitative theory of differential equations has been developed rapidly in the last decades, and there has been a great deal of works on the oscillatory behavior of integer order differential equations [1] [2] [3]. As a new cross-cutting area, recently some attention has been paid to oscillations of fractional differential equations [4] [5] [6] [7]. Some new developments in the oscillatory behavior of solutions of fractional differential equations with damping terms [8] [9] [10] [11] have been reported by authors.

In this paper, we consider the oscillatory behavior of solutions of the following fractional differential equation: 


$$
\begin{aligned}
& \frac{\partial}{\partial t}\left[a(t) g\left(p(t)+q(t) \mathrm{D}_{+, t}^{\alpha} u(x, t)\right)\right]+\sum_{i=1}^{m} a_{i}(x, t) f_{i}\left(\int_{0}^{t}(t-s)^{-\alpha} u(x, s) d s\right) \\
& =b(t) h(u) \Delta u(x, t)+\sum_{i=1}^{m} b_{i}(t) h_{i}\left(u\left(x, t-\tau_{i}\right)\right) \Delta u\left(x, t-\tau_{i}\right), t \geq t_{0}>0
\end{aligned}
$$

where $(x, t) \in \Omega \times R_{+}=E, \Delta$ is the Laplacian in $R^{n}, \Omega$ is a bounded domain in $R^{n}$ with a piecewise smooth boundary $\partial \Omega .0<\alpha<1$ is a real number and $D_{+}^{\alpha} u(x, t)$ is the Riemann-Liouville left-sided fractional derivative of order $\alpha \in(0,1)$ of $u$ for $t \in R_{+}:=(0, \infty)$.

We shall consider Robin and Dirichlet boundary conditions

$$
\frac{\partial u(x, t)}{\partial N}+\gamma(x, t) u(x, t)=0, \quad(x, t) \in \partial \Omega \times R_{+},
$$

and

$$
u(x, t)=0, \quad(x, t) \in \partial \Omega \times R_{+} .
$$

where $\gamma \in C\left[\partial \Omega, R_{+}\right]$is continuous function, $N$ is the unit out normal vector to $\partial \Omega$.

The following conditions are assumed to hold:

(H1) $f_{i, g} g \in C(R ; R)$ are convex in $[0, \infty)$ and $g$ is a monotone increasing function with $x f_{i}(x)>0, x g(x)>0$ for $x \neq 0$, there exist positive constants $k_{i}$, $\beta$ such that $f_{i}(x) / x>k_{i}, x / g(x)>\beta$ for $x \neq 0$. And $\tau_{i} \geq 0, u \neq 0$, $h(u)>0, \quad h_{i}(u)>0, u h^{\prime}(u)>0, u h_{i}^{\prime}(u)>0, h(0)>0, h_{i}(0)>0$.

(H2) $a, a_{i}, b, b_{i}$ and $q$ are positive continuous functions on $t \in\left[t_{0}, \infty\right)$ for a certain $t_{0}>0$, and $p$ is a nonpositive continuous function on $t \in\left[t_{0}, \infty\right)$ for a certain $t_{0}>0$. There exists a constant $M>0, q(t) \leq M$ for $t_{0}>0$. And $\left(\frac{-p(t)}{q(t)}\right)^{\prime} \neq 0, \quad t \in\left[t_{0}, \infty\right), \int_{t_{0}}^{\infty} \frac{-p(t)}{q(t)} d t<\infty$.

(H3) $g^{-1} \in C(R ; R)$ is continuous function with $s g^{-1}(s)>0$ for $s \neq 0$, there exists positive constant $\delta$ such that $g^{-1}(u v) \leq \delta g^{-1}(u) g^{-1}(v)$ for $u v<0$, and $g^{-1}(u v) \geq \delta g^{-1}(u) g^{-1}(v)$ for $u v>0$.

(H4) $a_{i} \in C\left(\bar{E} ; R_{+}\right)$, and $a_{i}(t)=\min _{x \in \bar{\Omega}} a_{i}(x, t)$.

By a solution of (1.1), (1.2) and (1.3), it mean a nontrivial function $u \in C^{1+\alpha}\left(\bar{E} ; R_{+}\right)$with $\int_{0}^{t} u(x, s)(t-s)^{-\alpha} d s \in C^{1}\left(\bar{E} ; R_{+}\right)$, $r(t) g\left(p(t)+q(t) D_{+, t}^{\alpha} u(x, t)\right) \in C^{1}\left(\bar{E} ; R_{+}\right)$satisfies (1.1) for $t>0$ on $E$ and the boundary conditions (1.2) and (1.3).

A solution $u$ of (1.1) is said to be oscillatory if it is neither eventually positive nor eventually negative, otherwise it is nonoscillatory. Equation (1.1) is said to be oscillatory if all its solutions are oscillatory.

\section{Preliminaries}

In this section, there are several kinds of definitions of fractional derivatives and integrals and some lemmas which are useful throughout this paper.

Definition 2.1 [12] The Liouville left-sided fractional integral of order $\alpha>0$ 
of a function $f: R_{+} \rightarrow R$ on the half-axis $R_{+}$is given by

$$
\left(I_{0+}^{\alpha} f\right)(t)=\frac{1}{\Gamma(\alpha)} \int_{0}^{t}(t-v)^{\alpha-1} f(v) d v
$$

provided that the left side is pointwise defined on $R_{+}$, where $\Gamma$ is the gamma function.

Definition 2.2 [12] The Riemann-Liouville fractional partial derivative of order $0<\alpha<1$ with respect to $t$ of a function $u(x, t)$ is given by

$$
\left(D_{+}^{\alpha} u\right)(x, t)=\frac{\partial}{\partial t} \frac{1}{\Gamma(1-\alpha)} \int_{0}^{t}(t-v)^{-\alpha} u(x, v) d v .
$$

Lemma 2.3 [12] Let

$$
G(t)=\int_{0}^{t}(t-v)^{-\alpha} u(v) d v, \quad \alpha \in(0,1), \quad t>0
$$

Then

$$
G^{\prime}(t)=\Gamma(1-\alpha)\left(D_{+}^{\alpha} u\right)(t), \quad \alpha \in(0,1), \quad t>0
$$

Lemma 2.4 [4] If $X$ and $Y$ are nonnegative, then

$$
m X Y^{m-1}-X^{m} \leq(m-1) Y^{m}
$$

where the equality holds if and only if $X=Y$.

\section{Oscillation of (1.1) and (1.2)}

For the sake of convenience, we set

$$
\begin{gathered}
U(t)=\frac{1}{|\Omega|} \int_{\Omega} u(x, t) d x, \quad \text { where }|\Omega|=\int_{\Omega} d x \\
z(t)=p(t)+q(t)\left(D_{+}^{\alpha} U\right)(t)
\end{gathered}
$$

Theorem 3.1 Suppose that (H1)-(H5) hold and if the fractional differential inequality

$$
\frac{d}{d t}[a(t) g(z(t))]+\sum_{i=1}^{m} k_{i} a_{i}(t) G(t) \leq 0
$$

has no eventually positive solution and the fractional differential inequality

$$
\frac{d}{d t}[a(t) g(z(t))]+\sum_{i=1}^{m} k_{i} a_{i}(t) G(t) \geq 0
$$

has no eventually negative solution, every solution of (1.1) and (1.2) is oscillatory in $E$.

Proof Suppose that $u(x, t)$ is a nonoscillatory solution of (1.1) and (1.2), it is either eventually positive or eventually negative. Without loss of generality, we may assume that $u(x, t)$ is an eventually positive solution of (1.1) and (1.2) in $\Omega \times\left[t_{0}, \infty\right)$. Integrating (1.1) with respect to $x$ over $\Omega$, we obtain

$\int_{\Omega} \frac{d}{d t}\left[a(t) g\left(p(t)+q(t) \mathrm{D}_{+, t}^{\alpha} u(x, t)\right)\right] d x+\sum_{i=1}^{m} \int_{\Omega} a_{i}(x, t) f_{i}\left(\int_{0}^{t}(t-s)^{-\alpha} u(x, s) d s\right) d x$

$=b(t) \int_{\Omega} h(u) \Delta u(x, t) d x+\sum_{i=1}^{m} b_{i}(t) \int_{\Omega} h_{i}\left(u\left(x, t-\tau_{i}\right)\right) \Delta u\left(x, t-\tau_{i}\right) d x$.

Using Green's formula and boundary condition (1.2), it is obvious that 


$$
\begin{aligned}
& \int_{\Omega} h(u) \Delta u(x, t) d x=\int_{\partial \Omega} h(u) \frac{\partial u(x, t)}{\partial N} d s-\int_{\Omega} h^{\prime}(u)|\operatorname{grad} u|^{2} d x \\
&=-\int_{\partial \Omega} \gamma(x, t) u h(u) d s-\int_{\Omega} h^{\prime}(u)|\operatorname{grad} u|^{2} d x \leq 0, t \geq t_{1} \\
& \int_{\Omega} h_{i}\left(u\left(x, t-\tau_{i}\right)\right) \Delta u\left(x, t-\tau_{i}\right) d x \leq 0, \quad t \geq t_{1}
\end{aligned}
$$

By using Jensen's inequality and (H1), (H4), we get

$$
\begin{aligned}
& \int_{\Omega} a_{i}(x, t) f_{i}\left(\int_{0}^{t}(t-s)^{-\alpha} u(x, s) d s\right) d x \\
\geq & a_{i}(t) \int_{\Omega} d x f_{i}\left[\int_{0}^{t}(t-s)^{-\alpha}\left(\int_{\Omega} u(x, s) d x\left(\int_{\Omega} d x\right)^{-1}\right) d s\right] \geq k_{i} a_{i}(t) \int_{\Omega} d x G(t)
\end{aligned}
$$

Combining (3.3)-(3.6), we obtain

$$
\frac{d}{d t}[a(t) g(z(t))]+\sum_{i=1}^{m} k_{i} a_{i}(t) G(t) \leq 0
$$

Therefore, $U(t)$. is an eventually positive solution of (3.1), this contradicts the hypothesis.

Secondly, if $u(x, t)$ is an eventually negative solution of the problem (1.1) and (1.2), then using above procedure, we can easily show that

$U(t)=\frac{1}{|\Omega|} \int_{\Omega} u(x, t) d x$ is an eventually negative solution of the Equation (3.2).

This completes the proof.

Theorem 3.2 Suppose that (H1)-(H4) and

$$
\int_{t_{0}}^{\infty} g^{-1}\left(\frac{1}{a(t)}\right) d t=\infty
$$

hold. if there exists a positive function $r \in C^{1}\left[t_{0}, \infty\right)$ such that

$$
\limsup _{t \rightarrow \infty} \int_{t_{0}}^{t}\left[r(t) \sum_{i=1}^{m} k_{i} a_{i}(s)-\frac{M a(s)\left[r^{\prime}(s)\right]^{2}}{4 \beta \Gamma(1-\alpha) r(s)}\right] d s=\infty
$$

where $k_{i}, \quad \beta$ are defined as in (H1), then every solution of (3.1) and (3.2) is oscillatory.

Proof Suppose that $U(t)$ is a nonoscillatory solution of (3.1). Without loss of generality, we may assume that $U(t)$ is an eventually positive solution of (3.1). Then there exists $G(t)>0, t \in\left[t_{1}, \infty\right)$, where $G(t)$ is defined as in Lemma 2.3.

It follows from (3.7) that

$$
[a(t) g(z(t))]^{\prime} \leq-\sum_{i=1}^{m} k_{i} a_{i}(t) G(t) \leq 0, \quad t \in\left[t_{1}, \infty\right) .
$$

Thus, $a(t) g(z(t))$ is strictly decreasing on $a(t)>0$. Since $a(t)>0$ for $t \in\left[t_{1}, \infty\right)$ and (H1), we see that $z(t)$ is eventually of one sign. We claim that

$$
z(t)>0, \quad t \in\left[t_{1}, \infty\right) .
$$

If not, there exists $t_{2} \geq t_{1}$ such that $z\left(t_{2}\right)<0$. Since $a(t) g(z(t))$ is strictly decreasing on $\left[t_{1}, \infty\right)$ and it is clear that $a(t) g(z(t)) \leq a\left(t_{2}\right) g\left(z\left(t_{2}\right)\right)=c<0$, where $c$ is a constant for $t \in\left[t_{2}, \infty\right)$. Therefore, we have 


$$
z(t) \leq g^{-1}\left(\frac{c}{a(t)}\right)
$$

Due to $q(t)>0$ and $g^{-1}(c)<0$, we get

$$
\frac{z(t)}{q(t)}=\frac{p(t)}{q(t)}+D_{+}^{\alpha} U(t)<\frac{g^{-1}\left(\frac{c}{a(t)}\right)}{q(t)} \leq \frac{\delta g^{-1}(c) g^{-1}\left(\frac{1}{a(t)}\right)}{M},
$$

Integrating the above inequality from $t_{2}$ to $t$, from Lemma 2.3, we have

$$
\int_{t_{2}}^{t}\left(\frac{p(t)}{q(t)}+\frac{G^{\prime}(s)}{\Gamma(1-\alpha)}\right) d s<\int_{t_{2}}^{t} \frac{\delta g^{-1}(c) g^{-1}\left(\frac{1}{a(t)}\right)}{M} d s
$$

which yields

$$
G(t) \leq G\left(t_{2}\right)+\Gamma(1-\alpha)\left[\int_{t_{2}}^{t} \frac{-p(t)}{q(t)} d s+\frac{\delta g^{-1}(c)}{M} \int_{t_{2}}^{t} g^{-1}\left(\frac{1}{a(t)}\right) d s\right] .
$$

By (H2) and (3.8), letting $t \rightarrow \infty$, we get $\lim _{t \rightarrow \infty} G(t)=-\infty$. This contradicts the fact that $G(t)>0$. Hence, (3.11) holds.

From Lemma 2.3

$$
z(t)=p(t)+q(t)\left(D_{+}^{\alpha} U\right)(t)=p(t)+q(t) \frac{G^{\prime}(t)}{\Gamma(1-\alpha)},
$$

therefore,

$$
G^{\prime}(t)=\Gamma(1-\alpha) \frac{z(t)-p(t)}{q(t)} \geq \Gamma(1-\alpha) \frac{z(t)}{q(t)} \geq \Gamma(1-\alpha) \frac{z(t)}{M}
$$

Define the function $w(t)$ by the generalized Riccati substitution

$$
w(t)=r(t) \frac{a(t) g(z(t))}{G(t)}, \quad t \in\left[t_{1}, \infty\right) .
$$

Then we have $w(t)>0$ for $t \in\left[t_{0}, \infty\right)$, and from (3.18), it follows that

$$
\begin{aligned}
w^{\prime}(t) & =\left(\frac{r(t)}{G(t)}\right)^{\prime}[a(t) g(z(t))]+\frac{r(t)}{G(t)}[a(t) g(z(t))]^{\prime} \\
& \leq \frac{r^{\prime}(t)}{r(t)} w(t)-r(t) \sum_{i=1}^{m} k_{i} a_{i}(t)-\frac{\Gamma(1-\alpha) z(t)}{M} \frac{w(t)}{G(t)} \\
& \leq \frac{r^{\prime}(t)}{r(t)} w(t)-r(t) \sum_{i=1}^{m} k_{i} a_{i}(t)-\frac{z(t)}{g(z(t))} \frac{\Gamma(1-\alpha) w^{2}(t)}{M r(t) a(t)} \\
& \leq \frac{r^{\prime}(t)}{r(t)} w(t)-r(t) \sum_{i=1}^{m} k_{i} a_{i}(t)-\frac{\beta \Gamma(1-\alpha)}{M r(t) a(t)} w^{2}(t) .
\end{aligned}
$$

Taking

$$
m=2, X=\sqrt{\frac{\beta \Gamma(1-\alpha)}{M r(t) a(t)}} w(t), Y=\frac{1}{2} \sqrt{\frac{M r(t) a(t)}{\beta \Gamma(1-\alpha)}} \frac{r^{\prime}(t)}{r(t)}
$$

from Lemma 2.4 and (3.19), we get 


$$
w^{\prime}(t) \leq-r(t) \sum_{i=1}^{m} k_{i} a_{i}(t)+\frac{M a(t)\left[r^{\prime}(t)\right]^{2}}{4 \beta \Gamma(1-\alpha) r(t)},
$$

Integrating both sides of the inequality (3.21) from $t_{0}$ to $t$, and taking the limit supremum of both sides of the above inequality as $t \rightarrow \infty$, we get

$$
\limsup _{t \rightarrow \infty} \int_{t_{0}}^{t}\left[r(t) \sum_{i=1}^{m} k_{i} a_{i}(s)-\frac{M a(s)\left[r^{\prime}(s)\right]^{2}}{4 \beta \Gamma(1-\alpha) r(s)}\right] d s<w\left(t_{0}\right)<\infty .
$$

Which contradicts (3.9). The proof is complete.

Secondly, if $U(t)$ is an eventually negative solution of the fractional differential inequality (3.2) and there exists $G(t)<0, t \in\left[t_{1}, \infty\right)$. When (3.2) is oscillatory is similar to that of above procedure, and hence is omitted.

Theorem 3.3 Assume that (H1) - (H4) and (3.8) hold. Furthermore, suppose that there exist a positive function $r \in C^{1}\left[t_{0}, \infty\right)$ and a function $H \in C(D, R)$, where $D:=\left\{(s, t): s \geq t \geq t_{0}\right\}$, such that

$$
\begin{gathered}
H(t, t)=0, \quad \text { for } \quad t \geq t_{0} \\
H(s, t)=0, \quad \text { for } \quad(s, t) \in D_{0}
\end{gathered}
$$

where $D_{0}:=\left\{(s, t): s \geq t \geq t_{0}\right\}$ and $H$ has a nonpositive continuous partial derivative $H_{t}^{\prime}(s, t)=\partial H(s, t) / \partial t$ with respect to the second variable and satisfies

$$
\limsup _{s \rightarrow \infty} \frac{1}{H\left(s, t_{0}\right)} \int_{t_{0}}^{s} H(s, t)\left[r(t) \sum_{i=1}^{m} k_{i} a_{i}(t)-\frac{M a(t s)\left[r^{\prime}(t)\right]^{2}}{4 \beta \Gamma(1-\alpha) r(t)}\right] d t=\infty
$$

where $k_{i}, \quad \beta$ and $r(t)$ are defined as in Theorem 3.2. Then all solutions of (3.1) and (3.2) are oscillatory.

Proof Suppose that $U(t)$ is a nonoscillatory solution of (3.1). Without loss of generality, we may assume that $U(t)$ is an eventually positive solution of (3.1). We proceed as in the proof of Theorem 3.2 to get (3.21), Multiplying (3.21) by $H(s, t)$ and integrating from $t_{0}$ to $s$, for $s \in\left[t_{0}, \infty\right)$, we derive

$$
\begin{aligned}
\int_{t_{0}}^{s} H(s, t)\left[r(t) \sum_{i=1}^{m} k_{i} a_{i}(t)-\frac{M a(t s)\left[r^{\prime}(t)\right]^{2}}{4 \beta \Gamma(1-\alpha) r(t)}\right] d t & \leq-\left.[H(s, t) w(t)]\right|_{t_{0}} ^{s}+\int_{t_{0}}^{s} H_{t}^{\prime}(s, t) w(t) d t \\
& \leq H\left(s, t_{0}\right) w\left(t_{0}\right),
\end{aligned}
$$

Therefore,

$$
\frac{1}{H\left(s, t_{0}\right)} \int_{t_{0}}^{s} H(s, t)\left[r(t) \sum_{i=1}^{m} k_{i} a_{i}(t)-\frac{M a(t s)\left[r^{\prime}(t)\right]^{2}}{4 \beta \Gamma(1-\alpha) r(t)}\right] d t \leq w\left(t_{0}\right)<\infty,
$$

which is a contradiction to (3.23). The proof is complete.

Secondly, if $U(t)$ is an eventually negative solution of the fractional differential inequality (3.2). The proof when (3.2) is oscillatory is similar to that of above procedure, and hence is omitted.

Next, we consider the case

$$
\int_{t_{0}}^{\infty} g^{-1}\left(\frac{1}{a(t)}\right) d t<\infty
$$

which is different from (3.8). In this case, we have the following results. 
Theorem 3.4 Assume that (H1)-(H4) and (3.26) hold, and that there exist a positive function $r \in C^{1}\left(\left[t_{0}, \infty\right) ; R\right)$ such that (3.9) holds. If for every constant $T=\max \left\{t_{2}, t_{3}\right\}$, such that

$$
\int_{T}^{\infty}\left(\frac{1}{a(t)} \sum_{i=1}^{m} \int_{T}^{t} a_{i}(s) d s\right) d t=\infty
$$

Then every solutions $U(t)$ of (3.1) and (3.2) are oscillatory or satisfies $\lim _{t \rightarrow \infty} G(t)=0$ or $\lim _{t \rightarrow \infty} G^{\prime}(t)=0$, where $G(t)$ is defined as Lemma 2.3.

Proof Suppose that $U(t)$ is a nonoscillatory solution of (3.1). We may assume that $U(t)$ is an eventually positive solution of (3.1), proceeding as in the proof of Theorem 3.2 to get (3.10). Then there are two cases for the sign of $z(t)$.

When $z(t)$ is eventually positive is similar to that of Theorem 3.2, we get that every solution $U(t)$ of (3.1) is oscillatory.

If $z(t)$ is eventually negative, there exists $t_{1} \geq t_{0}$, such that $z(t)<0$ for $t \geq t_{1}$. From (3.16), therefore,

$$
G^{\prime}(t)<\Gamma(1-\alpha) \frac{-p(t)}{q(t)}
$$

Since $\left(\frac{-p(t)}{q(t)}\right)^{\prime} \neq 0$ and $\int_{t_{0}}^{\infty} \frac{-p(t)}{q(t)} d t<\infty$ holds, then we obtain

$$
\lim _{t \rightarrow \infty} \frac{-p(t)}{q(t)}=0 .
$$

Letting $t \rightarrow \infty$ in (3.28), we have

$$
\lim _{t \rightarrow \infty} G^{\prime}(t) \leq 0 .
$$

If $\lim _{t \rightarrow \infty} G^{\prime}(t)<0$, then there exists $t_{2} \geq t_{1}$ such that $G^{\prime}(t)<0$ for $t \geq t_{2}$. Thus, we get $\lim _{t \rightarrow \infty} G(t)=A \geq 0$ and $G(t) \geq A, t \geq t_{2}$. Now we claim that $A=0$. If not, that is $\stackrel{t \rightarrow \infty}{A}>0$, then from (3.10), we derive

$$
[a(t) g(z(t))]^{\prime} \leq-\sum_{i=1}^{m} k_{i} a_{i}(t) G(t) \leq-\sum_{i=1}^{m} k_{i} a_{i}(t) A, \quad t \in\left[t_{2}, \infty\right) .
$$

Integrating both sides of (3.31) from $t_{2}$ to $t$, we have

$$
a(t) g(z(t)) \leq a\left(t_{2}\right) g\left(z\left(t_{2}\right)\right)-A \sum_{i=1}^{m} k_{i} \int_{t_{2}}^{t} a_{i}(s) d s \leq-A \sum_{i=1}^{m} k_{i} \int_{t_{2}}^{t} a_{i}(s) d s, \quad t \in\left[t_{2}, \infty\right),
$$

where $a\left(t_{2}\right) g\left(z\left(t_{2}\right)\right)>0$, Hence, from (H2) and (3.32), we get

$$
\frac{z(t)}{q(t)}=\frac{p(t)}{q(t)}+\frac{G^{\prime}(t)}{\Gamma(1-\alpha)} \leq \frac{1}{M} g^{-1}\left(\frac{-A \sum_{i=1}^{m} k_{i} \int_{t_{2}}^{t} a_{i}(s) d s}{a(t)}\right) .
$$

Integrating both sides of (3.33) from $t_{3}$ to $t$, we obtain

$$
G(t) \leq G\left(t_{3}\right)+\Gamma(1-\alpha)\left[\int_{t_{2}}^{t} \frac{-p(t)}{q(t)} d s+\frac{\delta g^{-1}(-A)}{M} \int_{t_{2}}^{t} g^{-1}\left(\frac{\sum_{i=1}^{m} k_{i} \int_{t_{2}}^{t} a_{i}(s) d s}{a(t)}\right) d u\right]
$$


using $g^{-1}(-A)<0$ and (3.27), as $t \rightarrow \infty, \lim _{t \rightarrow \infty} G(t)=-\infty$. which contradicts the fact that $G(t)>0$. Therefore, we have $\stackrel{t \rightarrow \infty}{A}=0$, that is $\lim _{t \rightarrow \infty} G(t)=0$. The proof is complete.

Secondly, if $U(t)$ is an eventually negative solution of the fractional differential inequality (3.2). The proof when (3.2) is oscillatory is similar to that of above procedure, and hence is omitted.

Theorem 3.5 Assume that (H1) - (H4) and (3.26) hold, Let $r(t)$ and $H(s, t)$ be defined as in Theorem 3.3 such that (3.23) holds. Furthermore, assume that (3.27) holds for every $T=\max \left\{t_{2}, t_{3}\right\}$. Then every solutions $U(t)$ of (3.1) and (3.2) are oscillatory or satisfies $\lim _{t \rightarrow \infty} G(t)=0$ or $\lim _{t \rightarrow \infty} G^{\prime}(t)=0$, where $G(t)$ is defined as Lemma 2.3.

Proof Suppose that $U(t)$ is a nonoscillatory solution of (3.1). Without loss of generality, assume that $U(t)$ is an eventually positive solution of (3.1), and proceeding as in the proof of Theorem 3.2 to get (3.11), there are two cases for the sign of $z(t)$.

When $z(t)$ is eventually positive, the proof is similar to that of Theorem 3.3. $z(t)$ is eventually negative, the proof is similar to that of Theorem 3.4. Here we omitted it.

\section{Oscillation of (1.1) and (1.3)}

In the next we establish sufficient conditions for the oscillation of all solutions of (1.1), (1.3). For this we need the following:

The smallest eigenvalue $\lambda_{0}$ of the Dirichlet problem

$$
\begin{gathered}
\Delta w(x)+\lambda w(x)=0, \quad \text { in } \quad \Omega \\
w(x)=0, \quad \text { on } \quad \partial \Omega
\end{gathered}
$$

is positive and the corresponding eigenfunction $\phi$ is positive in $\Omega$.

Theorem 4.1 Let all the conditions of Theorem 3.2 and 3.3 be hold. Then every solution of (1.1) and (1.3) oscillates in $E$.

Proof Suppose that $u(x, t)$ is a nonoscillatory solution of (1.1) and (1.3). Without loss of generality, we may assume that $u(x, t)$ is an eventually positive solution of (1.1) and (1.3) in $\Omega \times\left[t_{0}, \infty\right)$ for $t_{0}>0$. Multiplying both sides of the Equation (1.1) by $\phi(x)>0$ and then integrating with respect to $x$ over $\Omega$, we obtain for $t>t_{1}$,

$$
\begin{aligned}
& \int_{\Omega} \frac{d}{d t}\left[a(t) g\left(p(t)+q(t) \mathrm{D}_{+, t}^{\alpha} u(x, t)\right) \phi \phi(x) d x+\sum_{i=1}^{m} \int_{\Omega} a_{i}(x, t) f_{i}\left(\int_{0}^{t}(t-s)^{-\alpha} u(x, s) d s\right) \phi(x) d x\right. \\
& =b(t) \int_{\Omega} h(u) \Delta u(x, t) \phi(x) d x+\sum_{i=1}^{m} b_{i}(t) \int_{\Omega} h_{i}\left(u\left(x, t-\tau_{i}\right)\right) \Delta u\left(x, t-\tau_{i}\right) \phi(x) d x .
\end{aligned}
$$

Using Green's formula and boundary condition (1.3), it is obvious that

$$
\begin{array}{r}
\int_{\Omega} \Delta u(x, t) \phi(x) d x=\int_{\Omega} u(x, t) \Delta \phi(x) d x=-\lambda_{0} \int_{\Omega} u(x, t) \phi(x) d x \leq 0, \quad t \geq t_{1} . \\
\int_{\Omega} \Delta u\left(x, t-\tau_{i}\right) \phi(x) d x=-\lambda_{0} \int_{\partial \Omega} u\left(x, t-\tau_{i}\right) \phi(x) d x \leq 0, \quad t \geq t_{1} \cdot(4.4)
\end{array}
$$


By using Jensen's inequality and (H1) and (H4), we get

$$
\begin{aligned}
& \int_{\Omega} a_{i}(x, t) f_{i}\left(\int_{0}^{t}(t-s)^{-\alpha} u(x, s) d s\right) \phi(x) d x \\
\geq & a_{i}(t) \int_{\Omega} \phi(x) d x f_{i}\left[\int_{0}^{t}(t-s)^{-\alpha}\left(\int_{\Omega} u(x, s) \phi(x) d x\left(\int_{\Omega} \phi(x) d x\right)^{-1}\right) d s\right] \\
\geq & k_{i} a_{i}(t) \int_{\Omega} \phi(x) d x G(t) .
\end{aligned}
$$

Set

$$
U(t)=\int_{\Omega} u(x, t) \phi(x) d x\left(\int_{\Omega} \phi(x) d x\right)^{-1}, \quad \text { where }|\Omega|=\int_{\Omega} \phi(x) d x
$$

Combining (4.2)-(4.5), we obtain

$$
\frac{d}{d t}[a(t) g(z(t))]+\sum_{i=1}^{m} k_{i} a_{i}(t) G(t) \leq 0
$$

The rest of the proof is similar to that of Theorems 3.2 and 3.3, and hence the details are omitted.

Theorem 4.1 Let the conditions of Theorem 3.4 hold. Then every solution $U(t)$ of (1.1) and (1.3) is oscillatory or satisfies $\lim _{t \rightarrow \infty} G(t)=0$ or $\lim _{t \rightarrow \infty} G^{\prime}(t)=0$, where $U(t)$ is defined as Lemma 2.3.

Theorem 4.2 Let the conditions of Theorem 3.5 hold; Then every solution $U(t)$ of (4.6) is oscillatory or satisfies $\lim _{t \rightarrow \infty} G(t)=0$ or $\lim _{t \rightarrow \infty} G^{\prime}(t)=0$, where $G(t)$ is defined as Lemma 2.3.

The proofs of Theorem 4.1 and 4.2 are similar to that of Theorems 3.2-3.5 and hence the details are omitted.

\section{Applications}

Example 1 Consider the fractional differential equation

$$
\begin{aligned}
& \frac{\partial}{\partial t}\left[t^{\frac{2}{3}}\left(-\frac{1}{t}+t^{-\frac{1}{2}} \mathrm{D}_{+, t}^{\alpha} u(x, t)\right)\right]+\left(x^{\frac{3}{2}}+\frac{8}{3} t^{\frac{5}{3}}\right) \int_{0}^{t}(t-s)^{-\alpha} u(x, s) d s=e^{-t} \Delta u(x, t) \\
& +\Delta u(x, t-\pi), t>0
\end{aligned}
$$

Here, $\quad a(t)=t^{\frac{2}{3}}, \quad p(t)=-\frac{1}{t}, \quad q(t)=t^{-\frac{1}{2}}, \quad b(t)=t^{\frac{2}{3}}, \quad a_{1}(x, t)=x^{\frac{3}{2}}+\frac{8}{3} x^{\frac{5}{3}}$ and $f(x)=g(x)=x$.

Taking $\tau_{i}=\pi, \Omega_{1}=(0, \pi), \quad m=1, t_{0}=1, \quad k_{1}=1, \quad \beta=4, \quad M=9$, $a_{1}(t)=\min _{x \in \bar{\Omega}} a_{1}(x, t)=t^{\overline{2}}$.

Then, we get

$\int_{t_{0}}^{\infty} g^{-1}\left(\frac{1}{a(t)}\right) d t=\int_{1}^{\infty} \frac{1}{t^{\frac{2}{3}}} d t<\infty,\left(\frac{-p(t)}{q(t)}\right)^{\prime}=-\frac{1}{2} t^{-\frac{3}{2}} \neq 0, \int_{t_{0}}^{\infty} \frac{-p(t)}{q(t)} d t=\int_{1}^{\infty} \frac{1}{t^{\frac{1}{2}}} d t<\infty$.

It is clear that conditions (H1) - (H4) and (3.1) hold. Furthermore, taking $r(t)=t^{\frac{4}{3}}$ 
$\limsup _{t \rightarrow \infty} \int_{t_{0}}^{t}\left[r(s) \sum_{i=1}^{m} k_{i} a_{i}(s)-\frac{M a(s)\left[r^{\prime}(s)\right]^{2}}{4 \beta \Gamma(1-\alpha) r(s)}\right] d s=\limsup _{t \rightarrow \infty} \int_{1}^{t}\left[\frac{8}{3} s^{3}-\frac{9 s^{\frac{2}{3}}\left(\frac{4}{3} s^{\frac{2}{3}}\right)^{2}}{16 \Gamma(1-\alpha) s^{\frac{4}{3}}}\right] d s=\infty$

which satisfies condition (3.10). For every constant $T \geq t_{0}, t \in[2 T, \infty)$, we obtain

$$
\int_{T}^{\infty}\left(\frac{1}{a(t)} \sum_{i=1}^{m} \int_{T}^{t} a_{i}(s) d s\right) d t=\int_{T}^{\infty}\left(\frac{1}{t^{\frac{2}{3}}} \int_{T}^{t} \frac{8}{3} s^{\frac{5}{3}} d s\right) d t \geq \int_{T}^{\infty}\left(\frac{1}{t^{\frac{2}{3}}} t^{\frac{8}{3}}\right) d t \rightarrow \infty, t \rightarrow \infty,
$$

Which shows that (3.27) holds. Therefore, by Theorem 3.4 every solution of (5.5) is oscillatory or satisfies $\lim _{t \rightarrow \infty} G(t)=0$ or $\lim _{t \rightarrow \infty} G^{\prime}(t)=0$.

\section{Acknowledgements}

This research was partially supported by grants from the National Basic Research Program of China, No. 41630643 and by the Science Foundation for The Excellent Youth Scholars of Ministry of Education of China, No. 11801530.

\section{Conflicts of Interest}

The authors declare no conflicts of interest regarding the publication of this paper.

\section{References}

[1] Yang J., Liu, A. and Liu, G. (2013) Oscillation of Solutions to Neutral Nonlinear Impulsive Hyperbolic Equations with Several Delays. Electronic Journal of Differential Equations, 2013, 207-211.

[2] Liu, A., Ma, Q. and He, M. (2010) Oscillation of Nonlinear Impulsive Parabolic Equations of Neutral Type. Rocky Mountain Journal of Mathematics, 36, 1011-1026. https://doi.org/10.1216/rmjm/1181069442

[3] Liu, A., Xiao, L., Liu, T. and Zou, M. (2007) Oscillation of Nonlinear Impulsive Hyperbolic Equation with Several Delays. Rocky Mountain Journal of Mathematics, 37, 1669-1684. https://doi.org/10.1216/rmjm/1194275940

[4] Xiang, S., Han, Z., Zhao, P. and Sun, Y. (2014) Oscillation Behavior for a Class of Differential Equation with Fractional-Order Derivatives. Abstract and Applied Analysis, 2014, Article ID: 419597. https://doi.org/10.1155/2014/419597

[5] Grace, S.R., Agarwal, R.P., Wong, P.J.Y. and Zafer, A. (2012) On the Oscillation of Fractional Differential Equations. Fractional Calculus and Applied Analysis, 15, 222-231. https://doi.org/10.2478/s13540-012-0016-1

[6] Han, Z., Zhao, Y., Sun, Y. and Zhang, C. (2013) Oscillation Theorem for a Kind of Fractional Differential Equations. Discrete Dynamics in Nature and Society, 2013, 216-219. https://doi.org/10.1155/2013/390282

[7] Wang, Y., Han, Z., Zhao, P. and Sun, S. (2014) On the Oscillation and Asymptotic Behavior for a Kind of Fractional Differential Equations. Advances in Difference Equations, 2014, 50. https://doi.org/10.1186/1687-1847-2014-50

[8] Yang, J., Liu, A. and Liu, T. (2015) Forced Oscillation of Nonlinear Fractional Differential Equations with Dampingterm. Advances in Difference Equations, 2015, 1. https://doi.org/10.1186/s13662-014-0331-4 
[9] Qi, C. and Huang, S. (2013) Interval Oscillation Criteria for a Class of Fractional Differential Equations with Damping Term. Mathematical Problems in Engineering, 2013, Article ID: 301085. https://doi.org/10.1155/2013/301085

[10] Zheng, B. (2013) Oscillation for a Class of Nonlinear Fractional Differential Equations with Damping Term. Journal of Advanced Mathematical Studies, 6, 107-115. https://doi.org/10.1155/2013/912072

[11] Prakash, P., Harikrishnan, S. and Benchohra, M. (2015) Oscillation of Certain Nonlinear Fractional Partial Differential Equation with Damping Term. Applied Mathematics Letters, 43, 72-79. https://doi.org/10.1016/j.aml.2014.11.018

[12] Kilbas, A.A.A., Srivastava, H.M. and Trujillo, J.J. (2006) Theory and Applications of Fractinal Differential Equations. North-Holland Mathematics Studies, 204. 